\title{
Misoprostol para Indução do Parto: Abordagem Farmacoepidemiológica e Avaliação do Impacto na Redução de Cesáreas
}

\section{Misoprostol for Labor Induction: Pharmacoepidemiological Approach and Evaluation of The Impact on Cesareans Delivery Reduction}

\section{RESUMO}

Objetivo: Avaliar o uso do misoprostol para a indução do trabalho de parto em gestantes atendidas em um hospital público do Estado do Paraná e analisar a redução da prevalência de cesáreas após a inserção deste medicamento no estabelecimento. Metodologia: Estudo observacional transversal, com abordagem descritiva e analítica por meio de dados clínicos e epidemiológicos de todas as gestantes atendidas no hospital maternidade, no período entre julho de 2015 e junho de 2017. A análise estatística foi realizada pelo software Epi-info 6.04®. A significância estatística foi aceita ao nível de $95 \%(p \leq 0,05)$. Resultados: Foram identificadas 362 prescrições destinadas a gestantes majoritariamente adultas jovens $(64,1 \%)$, casadas $(61,9 \%)$, brancas $(58,6 \%)$, com ensino fundamental incompleto $(16,0 \%)$, residentes em área urbana $(78,2 \%)$, primíparas $(60,8 \%)$, com 41 semanas de gestação $(40,9 \%)$. As gestantaes foram tratadas, predominantemente com um único comprimido de misoprostol, para a indicação principal de gestação prolongada. O parto vaginal foi obtido somente em $48,62 \%$ dos casos, sobretudo em não primíparas $(p=0,004)$. Nas gestantes com maior paridade a quantidade necessária de comprimidos de misoprostol para atingir o desfecho (parto vaginal), foi menor $(p=0,021)$. Conclusão: Ocorreu um discreto aumento no número de partos por cesárea no hospital, indicando que a disponibilidade do misoprostol na maternidade não ocasionou a redução de cesáreas.

\section{DESCRITORES}

Misoprostol. Indução do Parto. Parto Vaginal. Parto Cesárea. Farmacoepidemiologia.

\begin{abstract}
Objective: To evaluate the use of misoprostol for the induction of labor in pregnant women attended in a public hospital of the state of Paraná and investigate the reduction of the prevalence of cesarean sections after the insertion of this medication in the establishment. Methodology: Cross-sectional observational study with a descriptive and analytical approach using clinical and epidemiological data of all pregnant women attended at the maternity hospital between the period of July 2015 and June 2017. Statistical analysis was performed using the Epi-info $6.04 \circledast$ software. Statistical significance was accepted at the $95 \%$ level $(p<0.05)$. Results: A total of 362 prescriptions were identified, with most pregnant women being young (64.1\%), married $(61.9 \%)$, white $(58.6 \%)$, with incomplete primary education $(16.0 \%)$, urban residents $(78.2 \%)$, primiparous $(60.8 \%)$, with 41 gestational weeks $(40.9 \%)$. These women were treated predominantly with a single misoprostol tablet for the main indication of prolonged gestation. Vaginal delivery was obtained only in $48.62 \%$ of the cases, especially in multiparous women $(p=0,004)$. In pregnant women with the highest parity, the number of tablets necessary to reach the outcome was lower $(p=0.021)$. Conclusion: There was a slight increase in the number of cesarean deliveries in the hospital, indicating that the availability of misoprostol in the maternity did not lead to a reduction in cesarean sections.
\end{abstract}

\section{DESCRIPTORS}

Misoprostol. Labor Induction. Vaginal Delivery. Cesarean Delivery. Pharmacoepidemiology.

\footnotetext{
${ }^{1}$ Programa de Pós-Graduação em Saúde Coletiva, Departamento de Saúde Coletiva, Universidade Federal do Paraná (UFPR), Curitiba, Paraná, Brasil.

${ }^{2}$ Docente do Departamento de Saúde Coletiva, Laboratório de Saúde Pública e Ambiental da UFPR, Curitiba, Paraná, Brasil.
} 
$\mathrm{O}$ aumento significativo do número de cesáreas nos últimos tempos tem estimulado um maior interesse pelo uso de métodos efetivos e seguros para a indução do parto na gestante $^{1,2}$.

A indução do parto é recomendada com objetivo de reduzir a mortalidade perinatal e materna. A decisão de induzir exige uma complexa avaliação, envolvendo o histórico clínico da gestante e a saúde do feto, assim como a avaliação da matriz uterina. Em países desenvolvidos, $25 \%$ das gestantes têm seu parto induzido. Nos países em desenvolvimento, este número é menor, porém crescente ${ }^{1,2}$.

Os métodos farmacológicos são os mais utilizados para a indução do trabalho de parto. A indução pode ocorrer pelo uso de ocitocina ou de prostaglandinas, tendo como exemplo sintético destas últimas, o misoprostol ${ }^{3}$.

O misoprostol é um derivado da prostaglandina E1, desenvolvido por meio de síntese em 1973, com objetivo de tratar úlceras pépticas e duodenais ${ }^{4,5}$. Em 1991, quando teve seu uso descrito pela primeira vez para indução do parto com o feto vivo, a sua utilização para essa finalidade se popularizou, principalmente devido a sua termoestabilidade, facilidade de conservação, menor risco de efeitos colaterais e menor custo quando comparado às demais prostaglandinas ${ }^{2,4,5}$.

Atualmente, no Brasil, o misoprostol é um medicamento sujeito a controle especial de uso restrito a clínicas e hospitais autorizados pela vigilância sanitária. Seu uso é destinado para fins obstétricos com prescrição e acompanhamento médico, em local e instalações adequadas, garantindo sua segurança, eficácia e uso racional ${ }^{6}$.
No ano de 2000, o Colégio Americano de Ginecologia e Obstetrícia (ACOG) recomendou o uso do misoprostol vaginal $25 \mu \mathrm{g}$ para a indução do parto, contribuindo, com a sua aplicação na prática mundial obstétrica ${ }^{7}$. Em 2009, foi incluído na lista de medicamentos essenciais pela Organização Mundial da Saúde $(\mathrm{OMS})^{8}$, e na Relação Nacional de Medicamentos Essenciais do Ministério da Saúde (MS) no Brasil'.

Nesse sentido, o uso do misoprostol nos centros obstétricos para indução do trabaIho de parto, em gestações a termo com feto vivo, visa a reduzir o número de cesáreas².

O objetivo deste estudo foi verificar se houve alteração na prevalência de cesáreas após a disponibilização do misoprostol em uma maternidade pública do estado do Paraná.

\section{METODOLOGIA}

Trata-se de um estudo observacional com caráter descritivo e analítico, retrospectivo e transversal, que visa a caracterizar e avaliar uso do medicamento misoprostol para a indução do trabalho de parto em gestantes atendidas em um hospital e maternidade do estado do Paraná (população do estudo), e o possível impacto na redução de cesáreas.

O período considerado é de julho de 2015 a junho de 2017, nos 24 meses que sucederam a autorização do uso do medicamento no estabelecimento. O hospital em estudo é integrante da rede do Sistema Único de Saúde e abriga um centro obstétrico de referência para gestação de alto risco, atendendo cerca de 3.000 partos ao ano.

$\mathrm{Na}$ análise da taxa de sucesso (efeti- 
vidade) do uso do misoprostol para a indução do parto, foi considerado sucesso quando o desfecho foi o parto vaginal, e insucesso quando resultou em parto cesárea.

Os dados foram coletados de: 1) Formulário interno de solicitação de misoprostol: idade materna, idade gestacional (calculada a partir da ultrassonografia), justificativa de uso do medicamento (por meio da Classificação Internacional de Doenças - CID 10) e posologia. 2) Prescrições médicas: tempo de tratamento e uso de ocitocina. 3) Dados sociodemográficos: por meio do sistema informatizado utilizado no hospital: cor/raça, escolaridade, estado civil e local de residência; 4) Prontuário eletrônico: tipo de parto realizado, justificativa para realização de cesárea, sucesso com o uso do medicamento, gestações anteriores (antecedente de cesariana, partos normais e abortos), paridade, complicações no parto ou pós-parto, comorbidades maternas e outros métodos auxiliares utilizados.

O banco de dados foi armazenado no Microsoft Excel 2003® e submetido à análise estatística descritiva com o programa Epi-info $6.04 ®$.

Através de testes não paramétricos e o cálculo do $p$-valor, foram analisados os fatores associados a taxa de sucesso (desfecho parto vaginal), variável dependente, considerando como variáveis independentes: idade da paciente (teste Mann Whitney); raça/ cor (teste Qui quadrado); idade gestacional (teste Mann Whitney); número de gestações prévias (teste Qui quadrado); antecedentes de aborto (teste Qui quadrado); antecedentes de partos vaginais (teste Qui quadrado); quantidade de comprimido administrados - de 1 a 4 comprimidos (teste Qui quadrado) e uso de misoprostol com ocitocina para indução do parto vaginal (teste Qui quadrado). Também foi verificada a associação da paridade com a quantidade de comprimidos administrados e a taxa de sucesso (Coeficiente de Spearman). A falta de normalidade das variáveis do estudo foi comprovada pelo teste de Shapiro Wilk.

Para a análise do impacto da implantação do misoprostol na indução do parto vaginal e consequente redução do número de cesáreas, foi utilizado o teste t-Student, com o cálculo do p-valor. Foram consideradas estatisticamente significativas as relações entre variáveis em que o $p$-valor foi menor que $5 \%$ ( $p \leq 0,05$, nível de 95\%).

Para a produção dos gráficos, foi utilizado o software Graphpad prism 6®.

Este estudo obteve aprovação do Comitê de Ética em Pesquisa da Universidade Federal do Paraná - Setor de Ciências da Saúde, sob parecer de número 017569/2017. Não possui conflito de interesses e segue os preceitos da Resolução n 466/12 do ConseIho Nacional de Saúde.

\section{RESULTADOS}

No período considerado, o misoprostol foi administrado a um total de 717 gestantes no hospital maternidade. Destas, 362 receberam o medicamento para fins de indução do trabalho de parto $(50,49 \%), 283$ para aborto $(39,47 \%)$ e 72 para tratamento da hemorragia pós-parto $(10,04 \%)$.

As características sociodemográficas das gestantes que utilizaram o medicamento para a indução do parto no período estudado (Tabela 1) e a variáveis clínicas e farmacológicas dessas gestantes (Tabela 2 ) encontram-se expostas a seguir. 
Tabela 1. Variáveis sociodemográficas das gestantes tratadas com misoprostol, em um hospital maternidade do estado do Paraná, 2015-2017

\begin{tabular}{|c|c|c|}
\hline Variáveis & $\mathrm{n}$ & $\%$ \\
\hline \multicolumn{3}{|l|}{ Faixa etária (anos) } \\
\hline$<20$ (adolescentes) & 91 & 25,14 \\
\hline 20 - 34 (adultas jovens) & 232 & 64,09 \\
\hline$>34$ (adultas) & 39 & 10,77 \\
\hline \multicolumn{3}{|l|}{ Estado civil } \\
\hline Solteira & 109 & 30,11 \\
\hline Casada/união estável & 224 & 61,88 \\
\hline Viúva & 02 & 0,55 \\
\hline Divorciada & 04 & 1,10 \\
\hline $\mathrm{NI}$ & 23 & 6,35 \\
\hline \multicolumn{3}{|l|}{ Escolaridade (anos) } \\
\hline Analfabeta & 09 & 2,48 \\
\hline Alfabetizada & 17 & 4,69 \\
\hline Fundamental Incompleto & 58 & 16,02 \\
\hline Fundamental Completo & 27 & 7,46 \\
\hline Médio Incompleto & 41 & 11,32 \\
\hline Médio Completo & 57 & 15,75 \\
\hline Superior Incompleto & 07 & 1,93 \\
\hline Superior Completo & 05 & 1,38 \\
\hline $\mathrm{NI}$ & 141 & 38,95 \\
\hline \multicolumn{3}{|l|}{ Raça/Cor } \\
\hline Branca & 212 & 58,56 \\
\hline Parda & 135 & 37,29 \\
\hline Preta & 09 & 2,49 \\
\hline $\mathrm{NI}$ & 06 & 1,66 \\
\hline \multicolumn{3}{|l|}{ Local de Residência } \\
\hline Rural & 79 & 21,82 \\
\hline Urbana & 283 & 78,18 \\
\hline
\end{tabular}

$\mathrm{NI}$ : Não informado. Fontes: Formulário interno, sistema informatizado e prontuário eletrônico.

A posologia recomendada para a indução do trabalho de parto, segundo a American College of Obstetricians and Gynecologists (ACOG) em 2009, a OMS em 2011 e o MS, é de um comprimido de $25 \mu \mathrm{g}$ de misoprostol pela via vaginal, em intervalos de 6 horas, por até 48 horas $^{4,5,10}$. Caso o efeito desejado não ocorra, a dose pode ser aumentada após 24 horas de indução, para $50 \mu \mathrm{g}$ a cada 6 horas, até o máximo de 4 administrações ${ }^{5}$. Intervalos menores que 6 horas ocasionam efeitos aditivos e podem provocar hiperestimulação 
Tabela 2. Variáveis clínicas e farmacológicas das gestantes tratadas com misoprostol para indução do trabalho de parto, em um hospital maternidade do estado do Paraná, 2015-2017

\begin{tabular}{l|c|c}
\hline Variáveis Clínicas e Farmacológicas & $n=362$ & $\%$ \\
\hline Idade Gestacional (semanas) & & \\
\hline$<37$ & 15 & 4,14 \\
37 e 38 & 70 & 19,33 \\
39 e 40 & 125 & 34,53 \\
41 & 148 & 40,88 \\
$>42$ & 04 & 1,10 \\
\hline Paridade & & \\
\hline nenhuma & 220 & 60,77 \\
1 & 79 & 21,82 \\
2 & 35 & 9,67 \\
3 & 16 & 4,42 \\
4 & 09 & 2,49 \\
6 & 02 & 0,55 \\
8 & 01 & 0,26 \\
\hline Antecedentes de aborto & & \\
\hline Sim & 46 & 12,71 \\
Não & 316 & 87,29 \\
\hline Antecedentes de cesáreas & & \\
\hline Sim & 05 & 1,38 \\
Não & 357 & 98,62 \\
Quantidade de Comprimidos 25 mcg & & \\
\hline 1 & 224 & 61,88 \\
2 & 104 & 28,73 \\
3 & 26 & 7,18 \\
4 & 08 & 2,21 \\
\hline Uso de ocitocina complementar & 36 & 9,94 \\
\hline
\end{tabular}

Fontes: Formulário interno, prescrições médicas e prontuário eletrônico.

uterina, taquissistolia e sofrimento fetal ${ }^{4}$. A maioria das mulheres, $61,88 \%(n=224)$ utilizou somente um comprimido de $25 \mu \mathrm{g}$. Das 224 pacientes que utilizaram 1 comprimido para indução, 112 delas culminaram em parto vaginal, correspondendo a $30,94 \%$ do total (362). Destas, 3,86\% utilizaram também a ocitocina como método auxiliar. No estudo, a quantidade máxima administrada foi de 4 comprimidos de $25 \mu \mathrm{g}$ ( 1 comprimido a cada 6 horas), administrados a $2,21 \%$ das gestantes.

Com o propósito de analisar a taxa de sucesso com o uso do misoprostol para indução do trabalho de parto e o desfecho parto vaginal foi possível observar que não houve relação estatisticamente significativa com a idade da paciente $(p=0,067)$, raça/cor da paciente $(p=0,66)$, idade gestacional $(p=0,32)$, antecedentes de aborto $(p=0,72)$, ocorrência de partos vaginais prévios $(p=0,56)$, quantidade de comprimidos administrados (1 a 4 comprimidos) $(p=0,11)$ e o uso de misoprostol com ocitocina para indução $(p=1)$. Porém, foi significativa a comparação entre o número de gestações prévias e o sucesso da indução após tratamento com misoprostol $(p=0,0037)$, indicando que quanto maior o número de gestações prévias (sejam elas, 
Tabela 3. Variáveis relacionadas ao parto das gestantes tratadas com misoprostol, em um hospital maternidade do estado do Paraná, 2015-2017

\begin{tabular}{l|c|c}
\hline Variáveis & $\mathrm{n}$ & $\%$ \\
\hline Tipo de parto & \multicolumn{2}{l}{} \\
\hline Cesáreo & 186 & 51,38 \\
\hline Vaginal & 176 & 48,62 \\
\hline Métodos auxiliares do parto & 05 & 1,38 \\
\hline Vácuo-extrator & 06 & 1,66 \\
\hline Fórceps & 02 & 0,55 \\
\hline Krause & 02 & 0,55 \\
\hline Complicações pós-parto & 02 & 0,55 \\
\hline Histerectomia & 03 & 0,83 \\
Fratura da Clavícula do recém-nascido & 04 & 1,10 \\
\hline Sangramento moderado & 08 & 2,20 \\
\hline Retenção placentária & \multicolumn{2}{|l}{} \\
\hline Hemorragia
\end{tabular}

NI: Não informado

Fontes: Formulário interno e prontuário eletrônico.

Figura 1. Número de partos vaginais e cesáreos no período de janeiro de 2014 a junho de 2015 (prévio à introdução do misoprostol) e entre julho de 2015 a junho de 2017 (após a introdução do misoprostol), em um hospital maternidade do estado do Paraná, 2014-2017

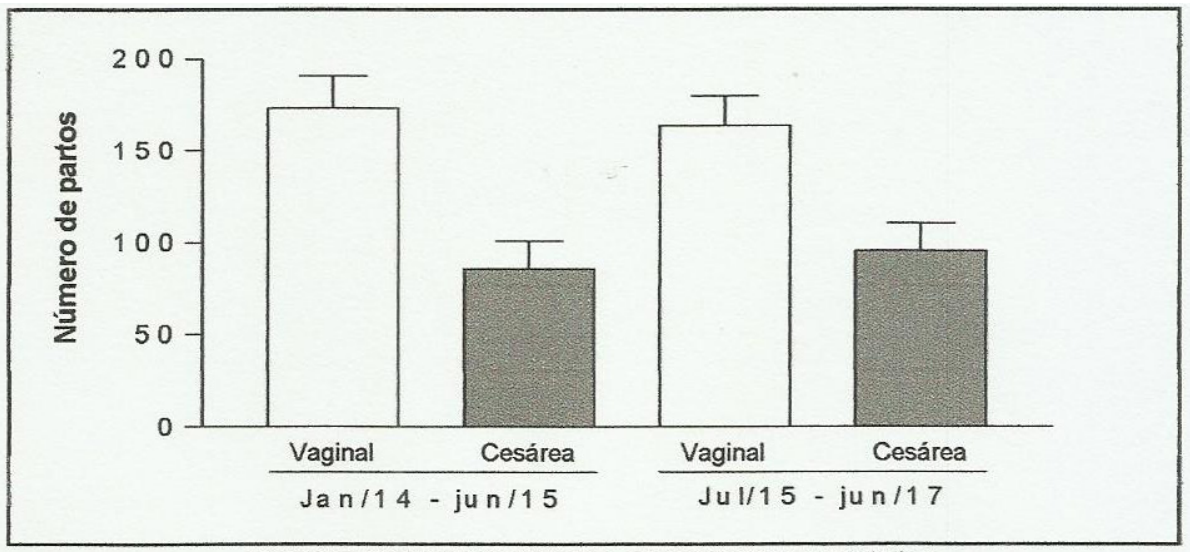

Fontes: Formulário interno e prontuário eletrônico 
Tabela 4. Análise do impacto na redução do número de cesáreas após a autorização do uso do misoprostol em um hospital maternidade do estado do Paraná, 2014-2017

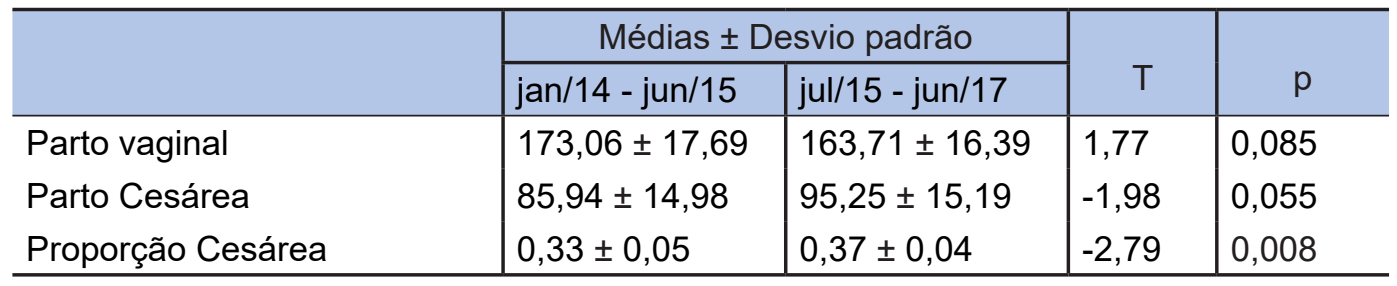

Fontes: Formulário interno e prontuário eletrônico

parto vaginal, cesárea ou aborto), maior a chance de sucesso. Este fato foi encontrado também com a quantidade de comprimidos administrados, indicando que quanto maior a paridade, menor o número de comprimidos necessários $(p=0,021)$ para atingir o desfecho do parto vaginal.

Constam 26 diferentes CIDs nas prescrições do misoprostol. O mais prescrito foi o O48 - "Gravidez prolongada" (35,64\%, $\mathrm{n}=129$ ), seguido do 042 - "Ruptura prematura de membranas" $(19,61 \%, n=71)$, do $0800-$ "Parto espontâneo cefálico" $(17,73 \%, n=64)$ e 013 "hipertensão gestacional” (6,09\%, n=22).

As variáveis relacionadas ao parto estão representadas na Tabela 3, na qual verificou-se que mais da metade das gestantes evoluiu para o parto cesárea $(51,38 \%)$, mesmo após o uso do medicamento.

Falha da indução $(48,39 \%, n=90)$ seguida das distócias de progressão - falta de dilatação, distócias cervicais e colo desfavorável $(11,82 \%, n=22)$; bradicardia fetal e desproporção cefalopélvica (cada uma com $6,99 \%, n=13$ ) e $25,81 \%$ de outras condições, foram justificativas para a realização do parto cesariano, mesmo após o uso do misoprostol, totalizando 24 justificativas para a ocorrência das 186 cesáreas.
Ao analisar as morbidades no decorrer da gestação até o nascimento dos filhos, foram identificadas 46 diferentes condições, numa frequência de 431 queixas relatadas. Neste valor considera-se que uma mesma gestante pode apresentar duas ou mais comorbidades durante todo o período gestacional. Dessas morbidades, a maior parte $(14,62 \%)$ se refere à hipertensão gestacional, e $14,39 \%$, a doenças do trato urinário. Quase $6 \%$ das gestantes permaneceram tabagistas durante a gestação, $4 \%$ foram diagnosticadas com sífilis e $1 \%$ não realizou as consultas do pré-natal. A presença de morbidades nas gestantes não teve impacto significativo na taxa de sucesso após o uso do medicamento $(p=0,24)$.

A fim de verificar o impacto da implantação do misoprostol na indução do parto vaginal e consequente redução do número de cesáreas, foram coletados dados de todos os partos realizados no hospital maternidade, de um ano e meio antes da introdução do misoprostol (de janeiro de 2014 a junho de 2015) e dos dois anos seguintes à introdução do medicamento (de julho de 2015 a junho de 2017), conforme demonstrado na Figura 1 e detalhado na Tabela 4.

Verificou-se um discreto aumento no 
número de partos por cesárea e redução do número de partos vaginais. Entretanto, estas modificações para cada tipo de parto ao longo do período compreendido, não foram estatisticamente significativas (Figura 1). Contudo, analisando a proporção de cesáreas em relação ao total de partos, observou-se um aumento significativo ao comparar os dois períodos $(p=0,008)$ (Tabela 4$)$.

\section{DISCUSSÃO}

A indução do trabalho de parto tem aumentado consideravelmente nos últimos anos no mundo, principalmente com a descoberta de novos métodos de indução ${ }^{5}$. A gestação prolongada e a ruptura prematura das membranas ovulares são as principais indicações para a indução do parto com o feto vivo, cujo propósito visa à redução das mortes e complicações perinatais e maternas, ${ }^{5,10,11,12,13}$. Neste estudo, a gestação prolongada foi a principal justificativa para indução do parto por meio da administração do misoprostol às gestantes.

Observou-se que $1,38 \%$ das gestantes receberam misoprostol, mesmo com antecedentes de cesariana, fato contraindicado pelas sociedades científicas $\mathrm{MS}^{2,9}, \mathrm{OMS}^{1}$ e $\mathrm{ACOG}^{10}$ devido ao aumento de chance de ruptura da cicatriz uterina e consequente possibilidade de hemorragia obstétrica, histerectomia e mortalidade materna e fetal. Entretanto, algumas revisões sistemáticas sobre a indução do trabalho de parto em mulheres com cesárea prévia demonstraram que os resultados obtidos de alguns ensaios clínicos randomizados analisados não foram conclusivos o suficiente para fundamentar de maneira irrefutável essa decisão clínica ${ }^{14,15}$. O MS, por sua vez, por meio da Portaria $n^{\circ}$ 306/2016, recomenda o uso do balão cervical ou da ocitocina para indução de trabalho de parto, quando este é indicado para mulheres com antecedentes de cesárea. Nesta situação, contraindica-se o uso do misoprostol.

A baixa efetividade do uso do misoprostol foi justificada principalmente devido à falha da indução. Menos da metade das gestantes com o parto induzido atingiram o parto vaginal $(48,62 \%)$ e $3 \%$ necessitaram do uso do vácuo-extrator ou do fórceps simpson para finalizar o procedimento. Porém, 61,88\% de todas as gestantes fizeram uso de somente um comprimido de $25 \mu \mathrm{g}, 9,94 \%$ delas utilizaram a ocitocina como medicamento auxiliar para indução, e menos de $1 \%$ utilizou o método de krause. Segundo a Febrasgo ${ }^{5}$, em torno de 4 a $10 \%$ das pacientes podem ter falha da indução pelo misoprostol. Nestas situações, este medicamento poderia ser utilizado por até $48 \mathrm{~h}$ (na posologia de 1 comprimido a cada 6 horas ou após 24 horas nesta posologia, ter um aumento para 2 comprimidos a cada 6 horas por mais 24 horas $)^{5}$. Se ainda assim não ocorrer o desfecho, é considerado falha da indução com o método (misoprostol), e deve ser iniciado o uso de ocitocina para dar continuidade à indução do trabalho de parto 5 . Somente após $12 \mathrm{~h}$ de indução com ocitocina e sem nenhuma ocorrência de complicação materna ou fetal, seria considerado falha de indução com necessidade de indicação de cesariana ${ }^{5}$.

Entretanto, a indução do parto deve ser realizada quando os possíveis benefícios se sobrepõem aos danos potenciais, considerando a real condição, desejos e preferências da gestante, além da avalição médica 
do estado cervical, do método de indução, das condições como paridade e ruptura de membrana. A indução deve ser realizada com cautela e sob vigilância com instalações disponíveis para avaliar o bem-estar materno e fetal, devido aos potenciais riscos de hiperestimulação uterina, ruptura de membrana e sofrimento fetal ${ }^{1}$.

McMaster et al. ${ }^{16}$ citam que a taxa de parto vaginal com uma única dose de misoprostol $25 \mu \mathrm{g}$, corresponde a aproximadamente $23 \%$. Comparativamente, após a administração da ocitocina, a taxa corresponde a $39,4 \%$.

No presente estudo foi encontrado que $30,94 \%$ das gestantes que utilizaram apenas um comprimido, culminaram para o parto vaginal, e somente $3,86 \%$ delas utilizaram a ocitocina como método auxiliar. PonMalar et al. ${ }^{17}$, por sua vez, compararam a administração do misoprostol $25 \mu \mathrm{g}$ em uma única dose com o placebo para a indução do parto em 126 gestantes, e obtiveram uma maior taxa de parto vaginal, estatisticamente significativa e mais rápida em comparação com aquelas que utilizaram o placebo $(p=$ $0,029)$, além de um decréscimo significativo no número de cesáreas nas mulheres tratadas com misoprostol ( $p=0.027)$. No atual estudo, das 224 gestantes que fizeram uso de apenas um comprimido de $25 \mu \mathrm{g}$ de misoprostol para a indução, quase metade atingiu o desfecho do parto vaginal, e as demais tiveram como justificativa a falha da indução. Dessa forma, a alta taxa de falha da indução relatada nos prontuários provavelmente não se justifica como real falha de indução, uma vez que não foram encontrados dados nos prontuários de maiores complicações maternas ou fetais que justificassem a realização do parto cesáreo.

$\mathrm{Na}$ literatura são enumerados vários motivos para a indicação do procedimento de cesárea. Dentre eles, a Febrasgo cita como absoluto: desproporção cefalopélvica, comprometimento anatômico do canal de parto, patologia anexial e sofrimento fetal agudo. Dentre as justificativas encontradas neste estudo, há um grande número de distócias maternas (falha de indução, distócia de progressão, colo desfavorável, distócia cervical e exaustão materna), o que por si só não teriam recomendação absoluta para a realização do parto cesárea ${ }^{18}$.

Quanto às condições clínicas observadas durante a gestação, dentre as analisadas, nenhuma delas parece interferir na ação do medicamento misoprostol de forma a ocasionar falha de indução e consequente indicação de cesariana ${ }^{5}$.A hipertensão arterial e pré-eclâmpsia, constam como as indicações mais comuns no Brasil para a realização de cesárea, porém, surpreendentemente, só apresentam respaldo científico se houver comprometimento fetal ou materno ${ }^{19}$.

Apesar do reconhecimento da importância clínica da cesariana, atualmente percebe-se uma provável banalização desse procedimento. Isso se confirma diante do fato de que o aumento da taxa de cesarianas não tem reduzido a mortalidade materna $e$ perinatal, além de ocasionar maiores gastos no sistema de saúde ${ }^{5}$.

Levando em consideração as características da população brasileira, a taxa de referência seria entre $25 \%-30 \%$ de procedimentos cesarianos no país, conforme instrumento desenvolvido pela $\mathrm{OMS}^{20}$. Entretanto os valores de cesárea no Brasil permanecem 
em torno de $55 \%$ desde 2010 . A região sul apresenta uma taxa ainda mais elevada de $60,5 \%$. Aproximadamente metade desta porcentagem de procedimentos cesáreos do país é realizada pela rede púbica de saúde ${ }^{21,22}$. Diante disto, o MS, a Febrasgo e o Conselho Federal de Medicina têm incentivado a realização do parto vaginal a fim de reduzir o número de cesáreas eletivas no Brasil, e, dentre as estratégias, encontra-se a indução do parto, com a utilização do misoprostol ${ }^{5}$.

Uma revisão sistemática estabeleceu que o misoprostol é mais efetivo do que o placebo, resultando em números menores de cesáreas quando comparado ao uso de outros uterotônicos (ocitocina e dinoprostone) e ao placebo ${ }^{23}$.

A prevalência de induções do parto é aproximadamente $20 \%$, porém essa taxa continua aumentando, pois tem sido cada vez mais empregada na obstetrícia, principalmente devido à disponibilidade de agentes farmacológicos para o amadurecimento cervical, com maior praticidade de uso (como o misoprostol e dinoprostone $)^{24,25}$. Assim, além do aumento de cesarianas, o aumento nas induções, também tem gerado uma preocupação obstétrica, e vários são os estudos com conclusões divergentes até o momento, indicando que em vez de reduzir os partos por cesarianas, a indução pode aumentá- $\operatorname{los}^{26,27}$ ou não interferir no desfecho ${ }^{28}$.

Um fator importante associado a uma maior probabilidade de cesariana após a indução do trabalho de parto é a idade materna avançada (> 35 anos), enquanto a alta paridade, o alto índice de Bishop e a maior duração do trabalho de parto apresentam menor probabilidade de parto cesáreo ${ }^{26,29}$.
No presente estudo, deve-se considerar que a não redução nos procedimentos cesarianos, pode ter ocorrido devido à resistência de administração contínua dos agentes de amadurecimento cervical quando não houve a progressão do parto de acordo com o esperado e à possibilidade de oferta de realização do parto cesárea à gestante em contrapartida a continuidade da indução do parto vaginal. Este fato é confirmado principalmente pela maioria das gestantes ter feito uso de somente um comprimido de misoprostol, quando poderiam ter sido administrados até oito comprimidos no período de 48h. Conforme descrito no documento "prevenção da primeira cesariana", raramente a falha da indução deveria ser justificativa para uma cesariana ${ }^{30,31}$.

Na literatura científica, vários estudos publicados consideram diferentes fatores associados, como idades gestacionais, paridades, idades e comorbidades maternas, entre outros, que podem influenciar no desfecho da indução e seu impacto nas cesáreas ${ }^{31}$. O atual estudo aborda, de maneira geral, todas as gestantes atendidas, com as mais diversas condições clínicas apresentadas durante a gestação, podendo ocasionar fatores de confundimento. Há, portanto, a necessidade de mais evidências que possam permitir a análise da influência de todas as variáveis que impactam na realização de cesarianas em gestantes com prognóstico à indução do parto induzido com misoprostol. Estas informações poderiam guiar de maneira mais efetiva os procedimentos, concentrando os esforços e os recursos nas gestantes com melhor previsão de resposta, além de minimizar sofrimento para a mãe e o concepto. 


\section{CONCLUSÃO}

Após a disponibilização do misoprostol na maternidade, observou-se o seu uso frequente para fins de indução do parto natural. Entretanto, obteve-se um resultado inverso, com discreto aumento no número de cesáreas. Portanto, o uso deste medicamento não ocasionou a redução de cesáreas esperada pelo serviço. Os resultados apontam para a necessidade de persistência na execução do protocolo, o qual requer administrações sucessivas do misoprostol a cada 6 horas ao longo de até 48 horas.

Neste sentido, quando a progressão do parto é lenta, torna-se mais atraente para a parturiente e para a equipe de saúde considerarem a realização do parto cesárea e justificarem esta escolha devido a uma suposta falha de indução. Corroborando, este estudo demonstrou que na maioria das gestantes foi administrado somente um comprimido de misoprostol.

A descrição clínica e epidemiológica do uso do misoprostol no presente estudo, fornece informações que podem auxiliar os gestores de saúde para o planejamento de ações e o desenvolvimento de programas e políticas estratégicas necessárias para garantir a implementação de intervenções eficazes. Estas intervenções poderiam reduzir a morbimortalidade materna e perinatal, o risco de internamentos prolongados e conseqüente aumento dos custos hospitalares, por permitir um adequado manejo na indução do trabalho de parto com o feto vivo. Além disso, esses dados podem servir como um espelho da qualidade do atendimento e das ações assistenciais prestadas às gestantes.

Deve-se considerar ainda que o crescente aumento de cesarianas é um grave problema de saúde pública, e o misoporostol se torna uma alternativa extremamente viável para a indução do parto vaginal com o feto vivo, devido ao seu baixo custo, facilidade de administração e estabilidade físico-química, quando comparado a outros medicamentos para a mesma finalidade. No entanto, o seu uso requer persistência da parturiente e o apoio da equipe de saúde, especialmente no primeiro e segundo estágios do trabalho de parto.

\section{REFERÊNCIAS}

1. OMS, Organização Mundial da Saúde. WHO recommendations for induction of labour. 2011 [acesso em 24 abr 2016].

2. Brasil. Ministério da Saúde. Secretaria de Políticas de Saúde. Área Técnica de Saúde da Mulher. Parto, aborto e puerpério: assistência humanizada à mulher. Brasília: 2001.

3. Alfirevic, Z, Keeney, E., Dowswell, T. et al. Which method is best for the induction of labour? A systematic review, network meta-analysis and cost-effectiveness analysis. Health Technol Assess. 2016;20(65):1-58.

4. Prostokos/misoprostol: comprimido vaginal. Cleverson Luiz dos Santos Vigo. Pernambuco: Hebron, 2001. Bula de remédio.

5. Febrasgo, Federação Brasileira das Associações de Ginecologia e Obstetrícia. Manual de Orientação Assistência

ao Abortamento, Parto e Puerpério. Comissões Nacionais Especializadas Ginecologia e Obstetrícia: 2010.

6. Brasil. Ministério da Saúde. Agência Nacional de Vigilância Sanitária. Portaria SVS/MS nº 06, de 29 de janeiro de 1999 Aprova a Instrução Normativa da Portaria SVS/MS n 344 de 12 de maio de 1998. Brasília: Anvisa; 1999

7. ACOG, American College of Obstetricians and Gynecologists Committee opinion - Induction of labor with Misoprostol. Int J Gynecol Obstet. 2000; 69:77-78.

8. OMS, Organização Mundial da Saúde. WHO model list of essential medicines. 16th list. March, 2009 [acesso em 25 abr 2016].

9. Brasil. Ministério da Saúde. Secretaria de Ciência, Tecnologia e Insumos Estratégicos. Formulário Terapêutico Nacional 2010: Rename 2010. $2^{\circ}$ edição. Brasília: 2010. 
10. ACOG, Colégio Americano de Ginecologia e Obstetrícia. Practice Bulletin $\mathrm{n}^{\circ}$. 107. Induction of labor. Obstet Gynecol. 2009; 114:386-97.

11. RCOG, Royal College of Obstetricians and Gynaecologists. RCOG evidence-based clinical guideline No. 9. Induction of labour. London (UK): RCOG Press; 2001.

12. Gülmezoglu AM, Crowther CA, Middleton P, Heatley E. Induction of labour for improving birth outcomes for women at or beyond term. Cochrane Database Syst Rev. 2012.

13. Mozurkewich E, Chilimigras J, Koepke E, Keeton K, King VJ. Indications for induction of labour: a best-evidence review. BJOG. 2009; 116(5):626-636.

14. Jozwiak M, Dodd JM. Methods of term labour induction for women with a previous caesarean section. Cochrane Database Syst Rev. 2013

15. Rath W, Tsikouras P. Misoprostol for Labour Induction after Previous Caesarean Section - Forever a "No Go"? Geburtshilfe und Frauenheilkunde. 2015; 75(11):1140-1147.

16. McMaster K, Sanchez-Ramos L, Kaunitz AM. Balancing the efficacy and safety of misoprostol: a meta-analysis comparing 25 versus 50 micrograms of intravaginal misoprostol for the induction of labour. BJOG. 2015; 122(4):468-476.

17. PonMalar J, Benjamin SJ, Abraham A, Rathore S, JeyaseeIan V. Randomized double-blind placebo controlled study of preinduction cervical priming with $25 \mu \mathrm{g}$ of misoprostol in the outpatient setting to prevent formal induction of labour. Arch Gynecol Obstet. 2017; 295:33-38.

18. FEBRASGO, Federação Brasileira das Associações de Ginecologia e Obstetrícia. Manual de orientação: assistência ao parto e tocurgia. $2002 ; 4: 48-49$.

19. Magee LA, Pels A, Helewa M. Rey E, Dadelszen PV. Diagnosis, evaluation, and management of the hypertensive disorders of pregnancy. J Obstet Gynaecol Can. 2008; 30(3): S1-48.

20. Brasil. Ministério da Saúde. Secretaria de Atenção à Saúde. Portaria n 306, de 28 de março de 2016. Aprova as Diretrizes de Atenção à Gestante: a operação cesariana. Brasília: Ministério da Saúde, 2016.

21. SINASC/SUS. Sistema de Informações sobre Nascidos Vivos. DATASUS. Ministério da Saúde; 2010. Ministério da Saúde; 2010 [acesso em 07 nov 2017]

22. SIH/SUS. Sistema de Internações Hospitalares. DATASUS. Ministério da Saúde; 2010 [acesso em 07 nov 2017].
23. Alfirevic Z Aflaifel N, Weeks A. Oral misoprostol for induction of labour. Cochrane Database of Systematic Reviews 2014 Issue 6. Art. No.: CD001338.

24. Goffinet F, Dreyfus M, Carbonne B, Magnin G, Cabrol D. Survey of the practice of cervical ripening and labour induction in France. J Gynecol Obstet Biol Reprod (Paris). 2003; 32:638-46.

25. Lyndon-Rochelle MT, Cárdenas V, Nelson JC, Holt VL, Gardella CMD, Easterling TR. Induction of labor in the absence of standard medical indications: incidence and correlates. Med Care 2007; 45:505-12.

26. Gerli S, Favilli A, Giordano C, Bini V, Di Renzo GCl. Single indications of induction of labor with prostaglandins and risk of cesarean delivery: A retrospective cohort study. J. Obstet. Gynaecol. Res. 2013; 39(5):926-931.

27. Jonsson M, Cnattingius S, Wikstrom AK. Elective induction of labor and the risk of cesarean section in low-risk parous women: a cohort study. Acta Obstet Gynecol Scand. 2013; 92:198-203

28. Gulmezoglu AM, Crowther CA, Middleton P, Heatley E. Induction of labour for improving birth outcomes for women at or beyond term. Cochrane Database Syst Rev. 2006; (4): CD004945

29. Roos N. Maternal risk factors for post term pregnancy and caesarean delivery following labour induction. Acta Obste Gynecol Scand. 2010; 89:1003-1010.

30. Spong CY, Berghella V, Wenstrom KD, Mercer BM, Saade GR. Preventing the first cesarean delivery: summary of a joint Eunice Kennedy Shriver National Institute of Child Health and Human Development, Society for Maternal-Fetal Medicine and American College of Obstetricians and Gynecologists Workshop. Obstet Gynecol. 2012; 120:1181-193.

31. Caughey $A B$. Induction of labour: does it increase the risk of cesarean delivery? BJOG. 2014;121(6):658-661.

\section{CORRESPONDÊNCIA}

Daeska Marcella Koch

Universidade Federal do Paraná

Laboratório de Saúde Pública e Ambiental,

Curso de Farmácia, Departamento de Saúde Coletiva

Avenida Lothário Meissner, 632, Jardim Botânico,

CEP: 80210-170 - Curitiba (PR), Brasil

E-mail: daeka@hotmail.com 